\title{
A Potential Invasion-Promoting Role for Ezrin in Endometrial Carcinoma
}

\author{
Ahmed R. H. Ahmed ${ }^{1}$, Ghada M. Yousef ${ }^{2}$, Mohamed S. E. Abdel-Raheem ${ }^{3}$, \\ Eman M. S. Muhammad ${ }^{1}$
}

${ }^{1}$ Department of Pathology, Faculty of Medicine, Sohag University, Sohag, Egypt; ${ }^{2}$ Department of Pathology, Sohag Cancer Center, Sohag, Egypt; ${ }^{3}$ Department of Gynecology and Obstetrics, Faculty of Medicine, Sohag University, Sohag, Egypt

\begin{abstract}
Background: Ezrin is a cell membrane-cytoskeleton linker that is essential to maintain the normal cell shape and the integrity of cell-cell adhesion. Overexpression of ezrin is correlated with poor prognosis in several malignancies.

Aim: To evaluate the expression of ezrin in hyperplastic and neoplastic endometrial tissues and to correlate its expression with the clinical and pathological parameters of endometrial carcinoma.

Methods: Tissue sections of 66 specimens including 37 endometrial carcinoma, 16 atypical endometrial hyperplasia and 13 benign endometrial hyperplasia were evaluated for ezrin expression by immunohistochemistry.

Results: Ezrin expression was detected in all endometrial carcinoma specimens and in $90 \%$ of hyperplasia. There was redistribution of ezrin from membranous expression in endometrial hyperplasia to diffuse cytoplasmic expression in endometrial carcinoma $(\mathrm{p}<0.0001)$. Expression of ezrin was significantly higher in atypical compared to benign hyperplasia $(p<0.001)$ and it was relatively higher in endometrial carcinoma compared to atypical endometrial hyperplasia $(\mathrm{p}=0.086)$. Among the carcinoma specimens, expression of ezrin was significantly associated with invasion of myometrium $(\mathrm{p}=0.001)$, higher FIGO stage $(\mathrm{p}=0.008)$ and the presence of vascular tumor emboli $(\mathrm{p}=0.001)$. Muscleinvasive tumor cells expressed significantly higher levels of ezrin compared to non-invasive cells of the same tumor tissue $(\mathrm{p}<0.0001)$. Tumor size, tumor grade and villoglandular morphology did not correlate significantly with ezrin expression.

Conclusion: Ezrin was overexpressed in endometrial carcinoma and its expression was associated with the invasive potential of tumor cells.

Keywords: Carcinoma, Cytoplasmic Expression, Endometrium, Ezrin, Hyperplasia, Invasion Corresponding author: Dr. Ahmed R. H. Ahmed; Department of Pathology, Faculty of Medicine, Sohag University, Sohag, 82524, Egypt; Email: ahmed ahmed4@med.sohag.edu.eg

Submitted: 26-June-2020, Revised: 11-August-2020, Accepted: 22-August-2020, Published online: 29-August-2020
\end{abstract}

(cc) BY

\section{Introduction}

Ezrin is a member of Ezrin-Radixin-Moesin (ERM) family of proteins. These proteins are critical for the integrity and stability of surface epithelium. They regulate cell-cell and cell-matrix communications and adhesions through the organization of actin filaments and modification of cell membrane dynamics ${ }^{1}$. In addition, coupling of ERM molecules to trans-membrane receptors and downstream signaling components enables them to influence signal transduction of major cell functions including cell survival, apoptosis, migration and immune response ${ }^{2}, 3$. Mislocalization of ERM molecules can interfere with formation of receptor complexes that could affect cell adhesion and alter signaling pathways of growth factors ${ }^{1,2}$.

Ezrin is expressed as a single isoform in mammals with a structurally conserved $30 \mathrm{kDa}$ composite domain ${ }^{4}$. It is a cell membranecytoskeleton cross-linker that is enriched in microvilli and surface epithelia. The interaction between ezrin and actin cytoskeleton is required to establish and maintain normal cell shape and polarity ${ }^{5,6}$.

Several studies confirmed that ezrin binds to adhesion molecules such as CD43, CD44 and intercellular adhesion molecules; which are 
implicated in cell migration and tumor cell metastasis ${ }^{1,4}$.

Expression of ezrin protein was demonstrated in normal tissues at varying levels and it was detected in tissues and cell lines of several malignant tumors ${ }^{7}$. Its expression has been linked to poor prognosis of several neoplasms including mammary, ovarian, gastrointestinal, and lung carcinomas ${ }^{8,11}$. Despite numerous studies that focused on identifying the prognostic value of ezrin in solid tumors, the exact role of this protein in cancer progression in general is not fully understood and the evaluation of ezrin in endometrial carcinoma is particularly lacking.

This study was designed to investigate the expression of ezrin in pre-neoplastic and neoplastic endometrial lesions.

\section{Methods}

\section{Patients and Samples}

This retrospective study was conducted on archived paraffin-embedded samples of 66 patients at the Pathology Departments of the Faculty of Medicine - Sohag University and Sohag Oncology Center, Sohag, Egypt.

All patients had a prolonged history of irregular uterine bleeding and tissue specimens were obtained by dilatation and curettage in 15 (22.7\%) patients, subtotal hysterectomy in 7 (10.6\%) and radical hysterectomy with bilateral salpingooophrectomy in 44 (66.7\%).

\section{Immunohistochemistry}

Immuno-detection of ezrin protein was carried out using avidin / biotin peroxidase complex method.

The technique was performed at room temperature unless mentioned and all steps were separated by washing in phosphate buffer saline. Four micrometer-thick sections were deparaffinized, rehydrated and blocked for endogenous peroxidase activity by $\mathrm{H}_{2} \mathrm{O}_{2}$ solution $(0.5 \%)$. Antigens were unmasked by incubation in citrate buffer (10 mM, pH 6.0) for 10 minutes using a microwave at high power. The sections were incubated with super block (Lab Vision, Cat \# TP015-H) to reduce non-specific bindings; then incubated overnight with mouse monoclonal antiezrin antibody (Thermoscintific; Clone 3C12, Cat. \#MS-661-P0; $1: 100)$ at $4^{\circ} \mathrm{C}$. Next day, tissue sections were incubated with biotinylated secondary antibody (Thermoscintific, Cat. \#32020) for 30 minutes, followed by incubation with avidin / peroxidase complex reagent (Thermoscintific, Cat. \#32020) for 30 minutes and exposed to DAB (3,3'diaminobenzidine tetrahydrochloride) substrate for 10 minutes.

The sections were counterstained, dehydrated and mounted as usual. Sections of renal cell carcinoma served as positive controls for ezrin expression and replacement of primary antibody with washing buffer worked as negative control for immunohistochemistry steps.

The immune-stained sections were evaluated independently of clinicopathological data. Expression level of ezrin was measured by modified histoscore that combines intensity of immunoreactions with percentage of positive cells 12. Cells in four high power fields $(\mathrm{x} 400)$ were counted and scored in each case. The intensities of immunoreactions were stated as negative, weakly positive, moderately positive, or strongly positive that have been weighed as $0,1,3$, and 10 , respectively. The total score of each case was calculated by the following formula:

Histoscore $=\left(0 \times \frac{\mathrm{x}}{\%}\right.$ of cells with negative expression of ezrin $)+(1 \times \%$ of cells with mild expression of ezrin $)+(3 \times \%$ of cells with moderate expression of ezrin $)+(10 \times \%$ of cells with strong expression of ezrin)

Final histoscore falls between 0 (when all cells are negative) and 1000 (when all cells are strongly positive). In neoplastic lesions, invasive and noninvasive components of same tumor tissue were evaluated separately for ezrin expression. Cells present in two high power fields (x400) of each component were scored and calculated.

\section{Statistical Analyses}

Data were analyzed by IBM SPSS Statistics for Windows, Version 22.0 (Armonk, NY: IBM Corp., 2013) and statistical significance was stated as $\mathrm{p}<0.05$.

Categorical variables were expressed as numbers and percentages and continuous variables were expressed as means ( \pm standard deviation) or as medians (interquartile range [IQR]). The frequency of an observation among categories was measures by Chi-square test $\left(\chi^{2}\right)$ and the expression levels of ezrin among different types of hyperplasia, tumor grades and tumor stages were compared by either Mann-Whitney U or Kruskal Wallis test. 
The association of ezrin expression in invasive and non-invasive components of tumor tissue was measured by Spearman's correlation test and the expression levels among both components were compared by Wilcoxon signed rank test.

\section{Ethical Considerations}

This study was approved by the Research Ethics Committee of the Faculty of Medicine - Sohag University (code 2016).

\section{Results}

\section{Patient and Disease Characteristics}

The study included 66 specimens from female patients who aged between 40 and 80 years with a mean $( \pm$ SD) and median values of $56.4( \pm 9.4)$ and 56 years, respectively. About two thirds $(43 / 66,65 \%)$ of the patients were postmenopausal and the main presentation for all patients was abnormal uterine bleeding. Clinical and sonographic evaluations reported diffuse endometrial thickening in 31 (47\%) patients and uterine masses / polyps in 35 (53\%).

Malignant endometrial lesions were presented in 37 (56.1\%) cases while endometrial hyperplastic lesions were presented in 29 (43.9\%); of which 16/29 (55.2\%) were atypical hyperplasia and 13/29 (44.8\%) were benign hyperplasia.

Among the malignant lesions; the tumor diameter / endometrial thickness ranged between 1.5 and $8 \mathrm{~cm}$ with a mean $( \pm \mathrm{SD})$ value of 4.02 ( \pm 1.59) and a median value of $4 \mathrm{~cm}$. Histologically, all malignant lesions were endometrioid adenocarcinomas of which $10 / 37$ (27\%) had focal villoglandular morphology and 5/37 (13.5\%) showed focal squamous differentiation. The tumors were graded according the International Federation of Gynecology and Obstetrics (FIGO) grading system ${ }^{13}$ as I, II and III in 11/37 (29.7\%), 18/37 (48.6\%) and 8/37 (21.6\%) cases, respectively. According to revised FIGO staging system ${ }^{14}$; 19/37 (51.4\%) tumors were classified as FIGO IA while FIGO IB, II, IIIA and IIIB were recorded in 9, 5, 3 and 1 cases; respectively. Vascular tumor emboli were identified histologically in $7 / 37(18.9 \%)$ tumors.

\section{Profile and Distribution of Ezrin Expression}

Expression of ezrin was limited to glandular cell lining with negative expression in endometrial stromal cells and myometrium.

Ezrin molecule was detected in all (100\%) cases of neoplastic endometrial lesions and in 26/29 (90\%) of hyperplastic lesions. The expression was demonstrated as a brownish cytoplasmic and membranous staining (Figure 1) with a significant tendency at concentration to luminal border of endometrial glands in cases of endometrial hyperplasia compared to cytoplasmic expression in cases of endometrial carcinoma $\left[\chi^{2} \quad(1)=14.9\right.$, $\mathrm{p}<0.0001]$.

Tumor infiltrating lymphocytes showed moderate to strong expression of ezrin protein (Figure 1F). Ezrin histoscore ranged between 0 and 969 with a mean ( \pm SD) and median values of 396.3 $( \pm 270.4)$ and 325 , respectively.

\section{Association of Ezrin Expression with Progression of Endometrial Carcinoma}

In general, ezrin showed a significant higher expression in endometrial carcinoma compared to endometrial hyperplasia (Table 1, Man-Whitney, $\mathrm{p}<0.0001)$. The mean $( \pm S D)$ ezrin histoscore in endometrial carcinoma was 493.7 ( \pm 269.3) compared to $230.9 \quad( \pm 210.5)$ in endometrial hyperplasia.

Among hyperplastic lesions, expression of ezrin was significantly higher in atypical hyperplasia (Mann-Whitney, $\mathrm{p}<0.001$ ). Nonetheless, expression of ezrin was relatively higher in endometrial carcinoma compared to atypical endometrial hyperplasia (Figure 2, Mann-Whitney, $\mathrm{p}=0.086$ ).

In neoplastic endometrial tissue, ezrin was significantly higher in myometrium-invading tumors, in tumors with advanced FIGO stage and in tumors with histologically evident vascular invasion. Although ezrin expression showed no association with villoglandular morphology of tumor tissue; presence of squamous differentiation was significantly associated with reduced ezrin expression.

The expression of ezrin was not associated with tumor size, tumors gross type or tumor grade (Table 1).

Ezrin expression was evaluated in invasive and non-invasive tumor cells separately. The levels of ezrin expression in non-invasive tumor cells and in corresponding invasive cells were strongly associated (Figure 3A, Pearson's correlation, $\mathrm{P}<0.0001$ ). More importantly, tumor cells that invade myometrium expressed significantly higher levels of ezrin protein compared to non-invasive cells of same tumor tissue (Figure 3B). The median (IQR) of ezrin expression in invasive tumor cells was 681 (386-845) compared to 425 (238-696) in non-invasive tumor cells (Wilcoxon`s signed rank test, $\mathrm{p}<0.0001$ ). 


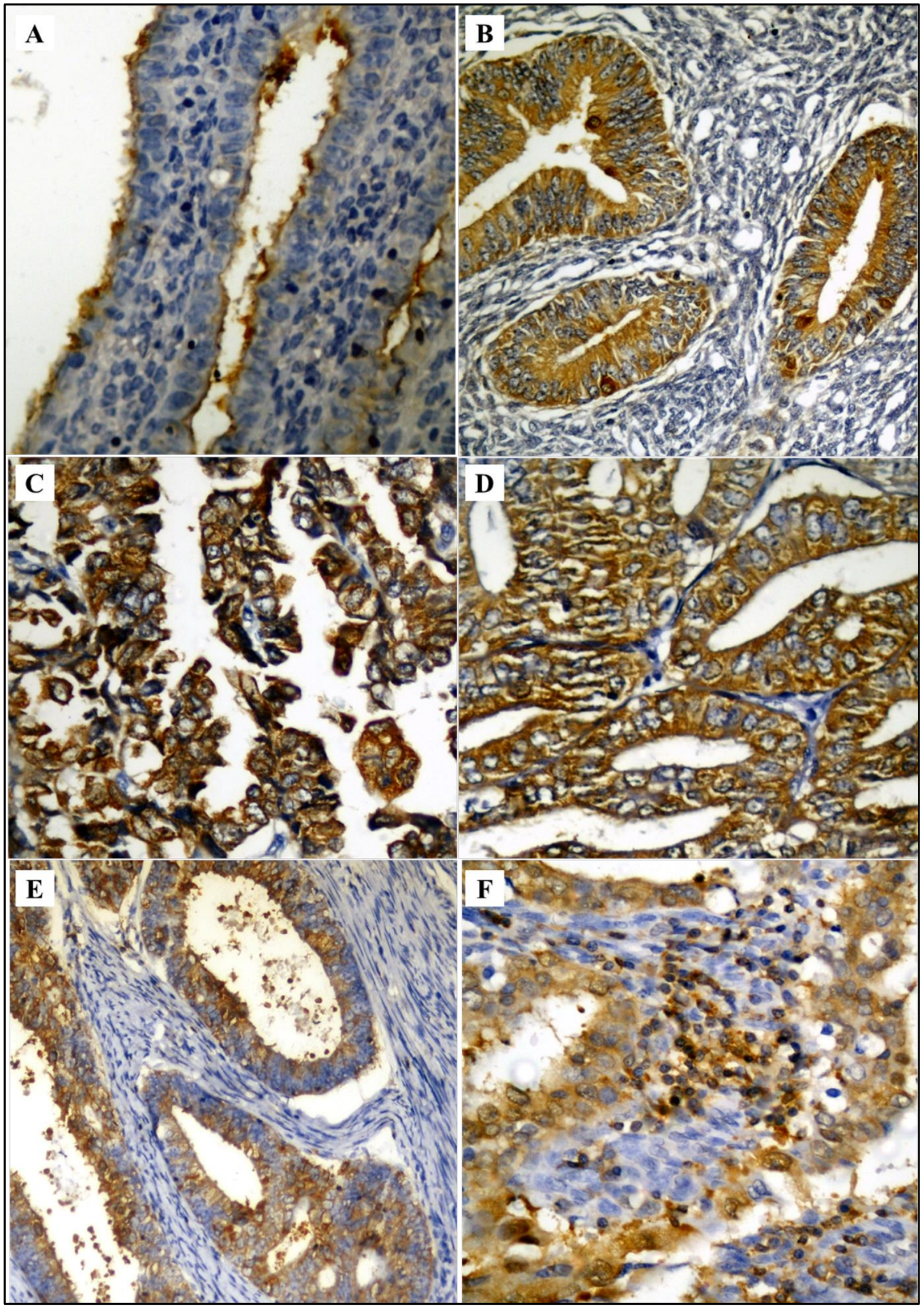

Figure 1: Membranous expression of ezrin in simple endometrial hyperplasia (A, $\mathrm{x} 400)$ versus membrano-cytoplasmic expression in atypical endometrial hyperplasia (B, $\mathrm{x} 400$ ), villoglandular carcinoma (C, x400) and endometrioid carcinoma (D, x400). Ezrin is strongly expressed by invasive glands $(\mathrm{E}, \mathrm{x} 200)$ and by tumor-infiltrating lymphocytes $(\mathrm{F}, \mathrm{x} 400)$ 
Table 1: Correlation of ezrin expression with different parameters of investigated lesions

\begin{tabular}{|c|c|c|c|c|}
\hline \multirow[t]{2}{*}{ Parameter } & & \multirow[t]{2}{*}{ No. (\%) } & Ezrin histoscore & \multirow[t]{2}{*}{ P-value } \\
\hline & & & Median (IQR) & \\
\hline \multirow[t]{2}{*}{ Benign vs. atypical hyperplasia } & Benign hyperplasia & $13(44.8)$ & $45(10-125)$ & \multirow{2}{*}{$<0.0001^{*}$} \\
\hline & Atypical hyperplasia & $16(55.2)$ & $350(201-470)$ & \\
\hline \multirow{2}{*}{ Hyperplasia vs. carcinoma } & Hyperplasia & $29(43.9)$ & $198(55-410)$ & \multirow{2}{*}{$<0.0001^{*}$} \\
\hline & Endometrial carcinoma & $37(56.1)$ & $480(267-741)$ & \\
\hline \multirow{2}{*}{$\begin{array}{l}\text { Atypical hyperplasia vs. } \\
\text { carcinoma }\end{array}$} & Atypical hyperplasia & $16(30.2)$ & $350(201-470)$ & \multirow[t]{2}{*}{$0.086^{*}$} \\
\hline & Endometrial carcinoma & $37(69.8)$ & $480(267-741)$ & \\
\hline \multirow[t]{2}{*}{ Pattern of carcinoma growth } & Diffuse endometrial growth & $10(27.0)$ & 677 (499-781) & \multirow[t]{2}{*}{$0.13^{*}$} \\
\hline & Localized uterine mass & $27(73.0)$ & $385(250-610)$ & \\
\hline \multirow[t]{2}{*}{ Tumor size } & $\leq 3 \mathrm{~cm}$ & $15(40.5)$ & $603(325-732)$ & \multirow[t]{2}{*}{$0.49^{*}$} \\
\hline & $>3 \mathrm{~cm}$ & $22(59.5)$ & $372(243-785)$ & \\
\hline \multirow[t]{2}{*}{ Villoglandular component } & Yes & $10(27.0)$ & $450(244-750)$ & \multirow[t]{2}{*}{$0.87^{*}$} \\
\hline & No & $27(73.0)$ & $480(285-750)$ & \\
\hline \multirow[t]{2}{*}{ Squamous differentiation } & Yes & $5(13.5)$ & $190(99-314)$ & \multirow[t]{2}{*}{$0.005^{*}$} \\
\hline & No & $32(86.5)$ & 549 (309-772) & \\
\hline \multirow[t]{3}{*}{ Grade } & 1 & $11(29.7)$ & $420(225-603)$ & \multirow[t]{3}{*}{$0.61^{* *}$} \\
\hline & 2 & $18(48.7)$ & $545(212-770)$ & \\
\hline & 3 & $8(21.6)$ & $493(329-795)$ & \\
\hline \multirow[t]{2}{*}{ Muscle invasion } & Yes & $28(75.7)$ & $606(335-795)$ & \multirow[t]{2}{*}{$0.001^{*}$} \\
\hline & No & $9(24.3)$ & $225(156-325)$ & \\
\hline \multirow[t]{2}{*}{ FIGO stage } & $\mathrm{I}$ & $28(75.7)$ & $355(221-608)$ & \multirow[t]{2}{*}{$0.008^{*}$} \\
\hline & II / III & $9(24.3)$ & $780(510-853)$ & \\
\hline \multirow[t]{2}{*}{ Detectable vascular tumor emboli } & Yes & 7 (18.9) & $876(800-924)$ & \multirow[t]{2}{*}{$0.001^{*}$} \\
\hline & No & $30(81.1)$ & 355 (224-605) & \\
\hline
\end{tabular}

*Mann-Whitney U test, **Kruskal-Wallis test; IQR: Interquartile range, FIGO: International Federation of Gynecology and Obstetrics

\section{Discussion}

Cell-cell and cell-matrix cross talks are essential to maintain normal function and morphology of surface epithelium. Ezrin-Radixin-Moesin proteins have been mainly concerned with epithelial morphogenesis, adhesion and migration ${ }^{1,6,15}$, three key events the disruption of which would participate in induction and progression of malignant tumors.

In this study, epithelial expression of ezrin was detected in all cases of endometrial carcinoma and in 26/29 cases of endometrial hyperplasia. However, ezrin expression was significantly higher in malignant compared to hyperplastic endometrial lesions $(p<0.0001)$. Nonetheless, expression of ezrin rose steadily from benign hyperplasia to atypical hyperplasia to malignant endometrial lesions. The three cases with negative ezrin expression and the four cases with low ezrin histoscore (less than 50) were benign hyperplastic lesions. When evaluated by Western blot analysis and immunehistochemistry in another study ${ }^{16}$, ezrin protein was higher in tissues of endometrial adenocarcinoma and atypical endometrial hyperplasia compared to normal endometrial tissue and simple endometrial hyperplasia. These findings imply a possible augmenting role of ezrin in early progression of endometrial carcinoma. This is supported by a recent report that activation of Ras oncogene requires ezrin participation and disrupting of interaction between ERM proteins and co-receptors abolishes Ras-induced cell growth ${ }^{17}$. Moreover, in vitro studies confirmed that ezrin was overexpressed and phosphorylated in ovarian cancer cell lines when stimulated by interleukin-1 alpha and EGF with subsequent significant stimulation of cell growth ${ }^{18}$.

In this study, the levels of ezrin expression in non-invasive and corresponding invasive tumor cells were strongly associated. Although this association was highly significant $(p<0.0001)$, it appeared that levels of ezrin molecule increased as the tumor cells infiltrate myometrium. In addition, expression of ezrin was higher in tumors with advanced FIGO stages that extended beyond uterine corpus and tumors with high ezrin levels had frequent vascular emboli. These observations suggest the emergence of clones of ezrin-expressing tumor cells that have a potentially higher invasive capacity. This claim is supported by overexpression 


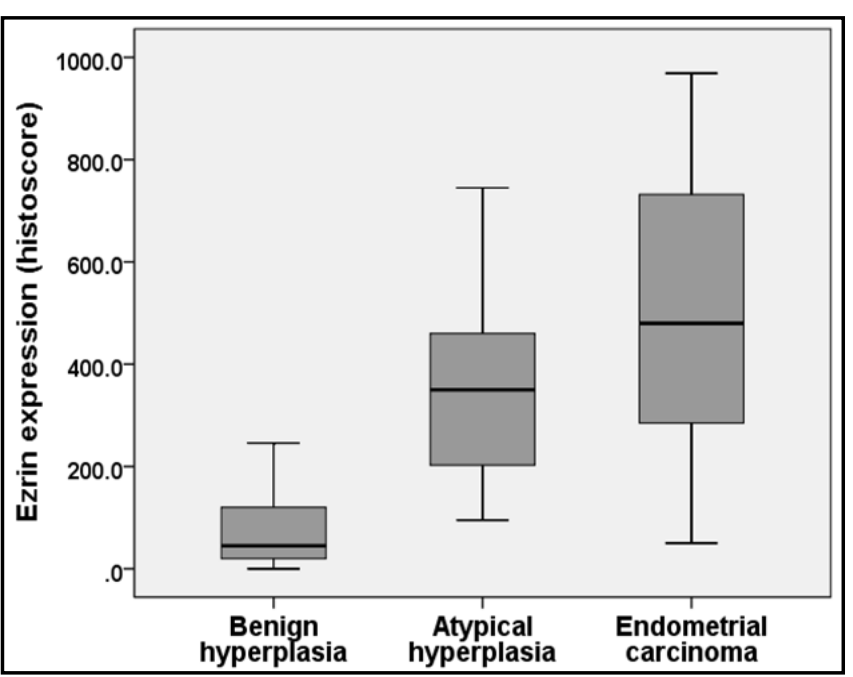

Figure 2: Expression level of ezrin in hyperplastic and neoplastic endometrial lesions

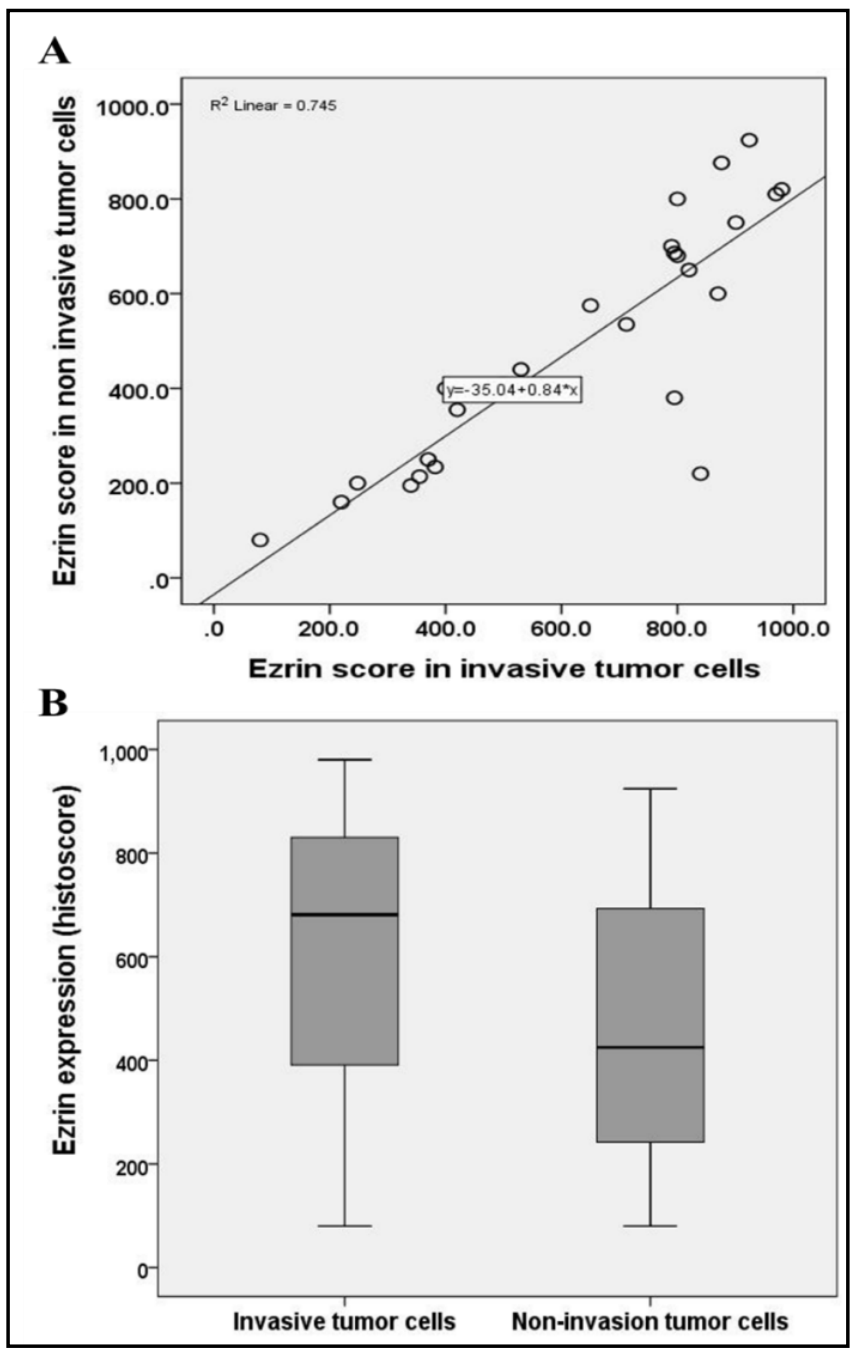

Figure 3: Expression of ezrin in invasive and non-invasive components of endometrial carcinoma (Although ezrin expression was strongly correlated in invasive and non-invasive tumor components [A], the histoscore was significantly higher in invasive tumors cells [B]) of ezrin in metastatic ovarian cancer cells compared to primary tumor cells ${ }^{18}$. In addition, Kong et al ${ }^{19}$ documented that the expression pattern of ezrin protein was closely related to lymphovascular invasion by malignant cells in cervical cancer and several other studies documented the strong association of high ezrin expression with raised metastatic potential of gynecological tumors including endometrial carcinoma ${ }^{20,21}$. In non-gynecological neoplasms, two separate meta-analyses including 2,701 and 6,675 patients showed that high levels of ezrin were associated with frequent nodal metastasis, distant metastasis and poor survival in several malignant tumors ${ }^{10,22}$.

Several in vitro studies have strongly suggested the invasion-promoting role of ezrin in malignant epithelial tumors. Inhibition of ezrin mRNA expression suppressed invasion of matrigel by endometrial carcinoma cell lines that are known to have high metastatic potential ${ }^{23}$. In addition, ezrin molecule potentiated invasiveness of ovarian carcinoma cell lines in chemo-invasion assay and inhibition of ezrin expression by sRNA reduced invasion capability of the cultured cells ${ }^{24}$. Similarly, ezrin overexpression induced cell protrusion, microvillus formation, migration and invasion of MiaPaCa-2 pancreatic cancer cell line while ezrin silencing induced opposite effects ${ }^{25}$.

Another interesting finding in the current study is the re-distribution of ezrin within malignant cells. Malignant endometrial glands showed cytoplasmic expression of ezrin compared to apical membranous expression in hyperplastic lesions $(p<0.001)$ which is compatible to the findings of Kobel et al ${ }^{20}$. They claimed that cells with intense cytoplasmic immunoreaction of ezrin lose their cell-cell contacts. In the same context, Ohtani et al ${ }^{16}$ have reported cytoplasmic localization of ezrin in most endometrial cancer cells and less frequently in endometrial hyperplastic cells. They emphasized that the expression and subcellular distribution of ezrin protein play an essential role in the development of endometrial carcinoma. In other gynecological tumors, homogenous cytoplasmic immunoreactivity of ezrin was demonstrated in serous ovarian carcinoma cells compared to apical expression in normal surface epithelial cells of the ovary ${ }^{26}$ and cytoplasmic localization of ezrin was associated with short overall survival of cervical carcinoma ${ }^{19}$.

Cytoplasmic localization of ezrin could provide a clue to understand the role of this molecule in 
progression of different epithelial tumors. The biological role or ezrin in tumor progression has been linked to its expression as well as subcellular localization within tumor cells ${ }^{4}$. Ezrin is normally synthesized as a dormant form that moves after activation from the peri-nuclear zone to the cell membrane where it binds to other members of ERM family and to target cytoskeleton molecules responsible for cell adhesion and cellular signaling. In malignant cells, ezrin is usually localized within the cytosol and hence cancer cells are easily detachable and more liable for epithelialmesenchymal transition with subsequent cell migration, invasion and metastasis ${ }^{1,}{ }^{19}$. This is compatible with findings of Pujuguet et al ${ }^{27}$ who concluded that ezrin molecule is critical for the transition of epithelial cells from a polarized closely adherent layer to a loosely attached spreading form by regulation of E-cadherin molecule in cell membrane.

\section{Conclusions}

Ezrin protein was overexpressed in endometrial carcinoma with cytoplasmic re-distribution of ezrin in malignant tumor cells.

Expression of ezrin was correlated with indicators of aggressive behavior of endometrial carcinoma; namely myometrial invasion, higher FIGO stages and penetration of lymphatic and vascular spaces.

Further evaluation of the relationship between ezrin and cell adhesion molecules as E-CadherinCatenin complexes and molecules involved in endometrial carcinogenesis as estradiol and PTEN may help in understanding the exact role of ezrin in endometrial carcinogenesis.

\section{Acknowledgement}

The authors thank the laboratory technicians at the Pathology Laboratory, Faculty of Medicine, Sohag University for the preparation of histological and immunestained tissue sections.

\section{Authors' contributions}

Conception or design: ARHA and MSEA; Acquisition, analysis or interpretation of data: All authors; Drafting the manuscript: ARHA and GMY; Revising the manuscript: MSEA and EMSM; Final approval of the manuscript version to be published: All authors; Agreement to be accountable for all aspects of the work: All authors.

\section{Conflict of interest}

The authors declare that they have no conflict of interest to disclose.

\section{Data a vailability}

Deidentified individual participant data used to produce the results of this study are available from the corresponding author (ARHA) upon request.

\section{Funding}

The authors did not receive funding for this study.

\section{Study registration}

None.

\section{References}

1. Arpin M, Chirivino D, Naba A, Zwaenepoel I. Emerging role for ERM proteins in cell adhesion and migration. Cell Adh Migr. 2011; 5(2): 199-206.

2. Fehon RG, McClatchey AI, Bretscher A. Organizing the cell cortex: the role of ERM proteins. Nat Rev Mol Cell Biol. 2010; 11(4): 276-287.

3. Pore D, Gupta N. The ezrin-radixin-moesin family of proteins in the regulation of B-cell immune response. Crit Rev Immunol. 2015; 35(1): 15-31.

4. Michie KA, Bermeister A, Robertson NO, Goodchild SC, Curmi PMG. Two sides of the coin: ezrin/radixin/moesin and merlin control membrane structure and contact inhibition. Int J Mol Sci. 2019; 20(8): 1996.

5. Clucas J, Valderrama F. ERM proteins in cancer progression. J Cell Sci. 2015; 128(6): 1253.

6. Kawaguchi K, Yoshida S, Hatano R, Asano S. Pathophysiological roles of ezrin/radixin/moesin proteins. Biol Pharm Bull. 2017; 40(4): 381-390.

7. Bruce B, Khanna G, Ren L, et al. Expression of the cytoskeleton linker protein ezrin in human cancers. Clin Exp Metastasis. 2007; 24(2): 69-78.

8. Ma L, Liu YP, Zhang XH, Geng CZ, Li ZH. Relationship of RhoA signaling activity with ezrin expression and its significance in the prognosis for breast cancer patients. Chin Med J (Engl). 2013; 126(2): 242-247.

9. Köbel M, Gradhand E, Zeng K, et al. Ezrin promotes ovarian carcinoma cell invasion and its retained expression predicts poor prognosis in ovarian carcinoma. Int J Gynecol Pathol. 2006; 25(2): 121-130.

10. Liang F, Wang Y, Shi L, Zhang J. Association of ezrin expression with the progression and prognosis of gastrointestinal cancer: a meta-analysis. Oncotarget. 2017; 8(54): 93186-93195.

11. Lee HW, Kim EH, Oh MH. Clinicopathologic implication of ezrin expression in non-small cell lung cancer. Korean J Pathol. 2012; 46(5): 470-477.

12. Ahmed ARH, Griffiths AB, Tilby MT, Westley BR, May FEB. TFF3 is a normal breast epithelial protein and is associated with differentiated phenotype in early breast cancer but predisposes to invasion and metastasis in advanced disease. Am J Pathol. 2012; 180(3): 904-916.

13. Gilks B. Uterus: Corpus. In: Rosai, Ackerman, eds. Surgical Pathology. $11^{\text {th }}$ ed. Philadelphia: Elsevier, 2018: 1294-355.

14. Pecorelli S. Revised FIGO staging for carcinoma of the vulva, cervix, and endometrium. Int J Gynaecol Obstet. 2009; 105(2): 103-104. 
15. Fiévet B, Louvard D, Arpin M. ERM proteins in epithelial cell organization and functions. Biochim Biophys Acta. 2007; 1773(5): 653-660.

16. Ohtani K, Sakamoto H, Rutherford T, et al. Ezrin, a membrane-cytoskeletal linking protein, is highly expressed in atypical endometrial hyperplasia and uterine endometrioid adenocarcinoma. Cancer Lett. 2002; 179(1): 79-86.

17. Sperka T, Geissler KJ, Merkel U, et al. Activation of Ras requires the ERM-dependent link of actin to the plasma membrane. PloS One. 2011; 6(11): e27511.

18. Chen Z, Fadiel A, Feng Y, Ohtani K, Rutherford T, Naftolin F. Ovarian epithelial carcinoma tyrosine phosphorylation, cell proliferation, and ezrin translocation are stimulated by interleukin 1alpha and epidermal growth factor. Cancer. 2001; 92(12): 3068-3075.

19. Kong J, Di C, Piao J, et al. Ezrin contributes to cervical cancer progression through induction of epithelialmesenchymal transition. Oncotarget. 2016; 7(15): 19631-19642.

20. Köbel M, Langhammer T, Hüttelmaier S, et al. Ezrin expression is related to poor prognosis in FIGO stage I endometrioid carcinomas. Mod Pathol. 2006; 19(4): 581-587.
21. Choi SD. Ezrin is an Essential Marker for Metastasis of Gynecologic Cancer. J Korean Soc Menopause. 2012; 18(2): 81-93.

22. Li J, Wei K, Yu H, Jin D, Wang G, Yu B. Prognostic value of Ezrin in various cancers: A systematic review and updated meta-analysis. Sci Rep. 2015; 5: 17903.

23. Ohtani K, Sakamoto H, Rutherford T, Chen Z, Satoh K, Naftolin F. Ezrin, a membrane-cytoskeletal linking protein, is involved in the process of invasion of endometrial cancer cells. Cancer Lett. 1999; 147(1-2): 31-38.

24. Horwitz V, Davidson B, Stern D, Tropé CG, Tavor Re'em T, Reich R. Ezrin is associated with disease progression in ovarian carcinoma. PloS One. 2016; 11(9): e0162502.

25. Meng Y, Lu Z, Yu S, Zhang Q, Ma Y, Chen J. Ezrin promotes invasion and metastasis of pancreatic cancer cells. J Transl Med. 2010; 8: 61.

26. Moilanen J, Lassus H, Leminen A, Vaheri A, Bützow R, Carpén O. Ezrin immunoreactivity in relation to survival in serous ovarian carcinoma patients. Gynecol Oncol. 2003; 90(2): 273-281.

27. Pujuguet P, Del Maestro L, Gautreau A, Louvard D, Arpin M. Ezrin regulates E-cadherin-dependent adherens junction assembly through Rac1 activation. Mol Biol Cell. 2003; 14(5): 2181-2191. 